\title{
The Steiner Problem for Infinitely Many Points
}

\author{
E. PAOLINI - L. ULIVI
}

ABSTRACT - Let $A$ be a given compact subset of the euclidean space. We consider the problem of finding a compact connected set $S$ of minimal 1-dimensional Hausdorff measure, among all compact connected sets containing $A$. We prove that when $A$ is a finite set any minimizer is a finite tree with straight edges, thus recovering the classical Steiner Problem. Analogously, in the case when $A$ is countable, we prove that every minimizer is a (possibly) countable union of straight segments.

\section{Introduction.}

Let $A=\left\{a_{1}, \ldots, a_{n}\right\}$ be a given set of points in $\mathbb{R}^{N}$. A natural task which arises in many optimization problems is that of finding a connected graph with minimal length whose vertices are the given points. If we are not allowed to add other vertices to the graph, the solution is called minimal spanning tree. Finding such a solution is a purely combinatorial problem. One can ambient the problem in the complete tree (i.e. the tree containing all possible edges with vertices in $A$ ) with weights on the ares given by the distance between the corresponding vertices. Then, one is requested to find a connected subgraph of the given weighted graph, which contains all the vertices and which has minimal length. It is clear that such a subgraph cannot contain loops (otherwise one can decrease the length by removing an arc in the loop) and hence it is actually a tree with $n$ vertices and $n-1$ arcs. It is not difficult to find an efficient algorithm to solve the minimal spanning tree problem (see [4, Section 2.4]). In this problem the geometry of the ambient space $\mathbb{R}^{N}$ has little relevance to the solution of the problem.

The problem becomes much more difficult if one has the possibility to add new vertices to the given set of points. This variant of the problem is

Indirizzo degli A.: Dip. Mat. "U. Dini”, Università di Firenze, Viale Morgagni 67/A 50134, Firenze. 
called Steiner problem. In other words the Steiner problem is that of finding a connected graph with minimal length among all graphs whose set of vertices contains the given set of points. The additional vertices are called Steiner points. It is easy to find examples where the addition of Steiner points is convenient. The simplest case can be found in the Fermat problem, which is the Steiner problem stated for three points: $A=\{a, b, c\}$. In this case one can prove that unless the triangle $a b c$ has an angle which is greater or equal to 120 degrees, it is convenient to add a Steiner point (called Fermat point) inside the triangle. By a variational argument it is easy to show that the Fermat point is characterized by having equal angles of 120 degrees between the three arcs joining the vertices of the triangle.

Since there is no a priori upper-bound on the number of vertices we can add, we are now faced with a problem in which an infinite number of graphs with different topologies should be considered. Moreover the position of Steiner points should be determined and hence it is an additional unknown to the problem. Fortunately also in this case it is possible to make an $a$ priori estimate on the number of vertices of the minimizer. So the problem reduces to a finite dimensional problem and existence of solutions can be achieved (see [4]). Notice, that such an existence result is not at all trivial, if approached from the graph theoretic setting. In fact the space of all finite graphs is dense in the space of all rectificable one-dimensional sets, hence one is requested to prove that a minimizing sequence converges to a finite graph.

Following these considerations one is naturally lead to state the Steiner problem in the more general framework given by Geometric Measure Theory. Instead of considering only finite graphs, we consider the family of all compact, connected sets $S$ containing a given compact set $A$. The length will be the 1-dimensional Hausdorff measure $\mathcal{H}^{1}$. The interest in this more general formulation is given by the recent development in the study of irrigation and urban planning problems which has originated from [3]. In particular in [5] the Steiner problem comes into play in this more general formulation.

The existence of minimizers, in this setting, is straighforward (Theorem 1.1). In [2] the existence result is proved in the general setting of metric spaces, using the results of [1]. In this paper (which originates from the Degree Thesis of the second author) we show that when $A \subseteq \mathbb{R}^{N}$ is finite, the minimizers are finite trees (Theorem 1.2). Thus we recover the equivalence with the original Steiner problem. As a consequence minimizers enjoy all the well known properties of minimal Steiner trees. We 
also show that when $A$ is countable the minimizers are countable trees (Theorem 1.6). This last result turns out to be much more difficult to prove, and needs some refined topological arguments.

In the last section we consider a more general problem, where we do not require $S$ to contain $A$ but we only require $S \cup A$ (together with $S$ ) to be connected. This allows one to consider sets $A \subseteq \mathbb{R}^{N}$ which can possibly have positive Lebesgue measure, without forcing $S$ to have infinite length. This setting was recently considered in [2] where some existence result are given in the more general case of metric spaces. In Theorem 2.1 we prove that in the case when $A$ has a finite number of connected components, then the minimizers are, again, finite graphs with straight edges.

As a final remark we notice that the Steiner problem can be settled in the framework of 1-dimensional minimal surfaces. In particular the theory developed by Reifenberg in [7] is very similar to the setting presented here. In fact Reifenberg considered compact sets to represent surfaces and used homological properties to fix the boundary condition. The more refined theory of varifolds is also able to deal with non-oriented surfaces. Applying to the Steiner problem the regularity results of these more general theories, we could obtain at once that the set $S$ is regular, and hence composed by straight segments, outside a closed singular set which is $\mathcal{H}^{1}$-negligible. In this paper we obtain more refined results which, of course, only apply to the 1-dimensional case.

The results of this paper have been subsequently extended and refined in the forthcoming paper [6].

\section{The general setting of the Steiner problem.}

Let $A$ be a compact subset of $\mathbb{R}^{N}$. We denote with

$$
\begin{aligned}
\mathcal{S}(A) & :=\left\{S \subseteq \mathbb{R}^{N}: S \text { is compact, connected and } \mathrm{S} \supseteq A\right\}, \\
\mathcal{M}(A) & :=\left\{S \in \mathcal{S}(A): \mathcal{H}^{1}(S) \leq \mathcal{H}^{1}\left(S^{\prime}\right), \forall S^{\prime} \in \mathcal{S}(A)\right\} .
\end{aligned}
$$

THEOREM 1.1 (existence of minimizers). Let $A$ be any compact subset of $\mathbb{R}^{N}$. Then $\mathcal{M}(A)$ is not empty.

Proof. Let

$$
\ell=\inf _{S \in \mathcal{S}(A)} \mathcal{H}^{1}(S)
$$


and let $R>0$ be such that $A \subseteq \overline{B_{R}(0)}$ (we denote with $B_{R}(x)$ the open ball centered in $x \in \mathbb{R}^{n}$ with radius $R>0$ ). If $\ell=+\infty$ then we have, trivially, $\overline{B_{R}(0)} \in \mathcal{M}(A)$. Suppose $\ell<+\infty$, and take any sequence $S_{k} \in \mathcal{S}(A)$ such that $\mathcal{H}^{1}\left(S_{k}\right) \rightarrow \ell$. Up to a subsequence we may suppose that $\mathcal{H}^{1}\left(S_{k}\right) \leq 2 \ell$ for all $k$. Moreover since every $S_{k}$ is connected, and contains at least one point of $B_{R}(0)$, we deduce that $S_{k} \subseteq \overline{B_{R+2 \ell}(0)}$ for all $k$. Hence by the compactness theorem of compact subsets of $\overline{B_{R+2 \ell}}$ we know that up to a subsequence $S_{k} \rightarrow S$ in the Hausdorff metric. It is easy to check that also $S \in \mathcal{S}(A)$. By Gołąb's Theorem (see [1]) we find that

$$
\mathcal{H}^{1}(S) \leq \liminf _{k} \mathcal{H}^{1}\left(S_{k}\right)=\ell
$$

and hence $S \in \mathcal{M}(A)$.

We introduce some notation. If $\gamma:[a, b] \rightarrow \mathbb{R}^{n}$ is a curve, we define:

$$
\begin{gathered}
{[\gamma]:=\gamma([a, b]), \quad(\gamma):=\gamma((a, b)), \quad[\gamma):=\gamma([a, b)), \quad(\gamma]:=\gamma((a, b]),} \\
\partial \gamma:=\{\gamma(a), \gamma(b)\} .
\end{gathered}
$$

If $x, y \in \mathbb{R}^{N}$ we define the closed and open segments with end-points in $x$ and $y$ :

$$
[x, y]:=\{t x+(1-t) y: t \in[0,1]\}, \quad(x, y):=\{t x+(1-t) y: t \in(0,1)\} .
$$

TheOREM 1.2 (Steiner tree over a finite set). Let $A=\left\{a_{1}, \ldots, a_{n}\right\}$ be $a$ subset of $\mathbb{R}^{N}$ and let $S \in \mathcal{M}(A)$.

Then there exists $m$ and points $x_{i}, y_{i} \in \mathbb{R}^{N}$ for $i=1, \ldots, m$ such that

$$
S=\bigcup_{i=1}^{m}\left[x_{i}, y_{i}\right]
$$

where the segments $\left[x_{i}, y_{i}\right]$ can only meet on their vertices, i.e. $\left[x_{i}, y_{i}\right] \cap$ $\cap\left(x_{j}, y_{j}\right)=\emptyset$ for all $i \neq j$.

Proof. First of all notice that $\mathcal{H}^{1}(S)<+\infty$, in fact if we consider

$$
\tilde{S}:=\bigcup_{i=1}^{n}\left[0, a_{i}\right]
$$

we notice that $\tilde{S} \in \mathcal{S}(A)$ and $\mathcal{H}^{1}(S) \leq \mathcal{H}^{1}(\tilde{S})<+\infty$.

Since $\mathcal{H}^{1}(S)<+\infty$ by [1, first rectifiability theorem] we know that given any two distinct points $x, y \in S$ there exists a lipschitz continuous injective curve $\gamma:[0,1] \rightarrow S$ such that $\gamma(0)=x, \gamma(1)=y$. 
For $j=2, \ldots, n$ consider the lipschitz continuous injective curves $\eta_{j}:[0,1] \rightarrow S$ such that $\eta_{j}(0)=a_{j}, \eta_{j}(1)=a_{1}$ and define

$$
\begin{aligned}
S_{1} & :=\left\{a_{1}\right\}, \\
S_{j} & :=\bigcup_{i=2}^{j}\left[\eta_{i}\right], \quad j=2, \ldots, n .
\end{aligned}
$$

Clearly $S_{n} \in \mathcal{S}(A)$ and by construction we have $S_{n} \subseteq S$. Now we claim that $S_{n}=S$. In fact suppose by contradiction that there exists $x \in S \backslash S_{n}$. Since $S_{n}$ is compact, for some $r>0$ we would have $S \backslash S_{n} \supseteq S \cap B_{r}(x)$. But $\mathcal{H}^{1}\left(S \cap B_{r}(x)\right) \geq r$ since $S$ is connected. Finally, recalling that $S_{n} \subseteq S$, we would find $\mathcal{H}^{1}(S)-\mathcal{H}^{1}\left(S_{n}\right) \geq \mathcal{H}^{1}\left(S \backslash S_{n}\right) \geq r>0$ which is a contradiction to the minimality of $S$.

Hence $S_{n}=S$. But in most cases the curves $\eta_{j}$ will have many points in common. First of all we consider the points

$$
t_{j}:=\min \left\{t \in[0,1]: \eta_{j}(t) \in S_{j-1}\right\}, \quad j=2, \ldots, n
$$

and consider the restricted curves $\tilde{\eta}_{j}=\left.\eta_{j}\right|_{\left[0, t_{j}\right]}$. Again we have that $S_{j}=S_{j-1} \cup\left[\tilde{\eta}_{j}\right]$ hence $S=S_{n}=\bigcup\left[\tilde{\eta}_{j}\right]$. Now the curves $\tilde{\eta}_{j}$ satisfy the property $\left(\tilde{\eta}_{j}\right) \cap\left[\tilde{\eta}_{i}\right]=\emptyset$ only when $j>i$ since in general the point $\eta_{j}\left(t_{j}\right)$ can be an internal point of some curve $\eta_{i}$ with $i<j$.

To get the result stated in the theorem we need to break each curve $\tilde{\eta}_{j}$ in many parts. First of all if $t_{j}=0$ we completely discard the curve $\tilde{\eta}_{j}$. Otherwise we consider the restrictions of the curve $\tilde{\eta}_{j}$ to the intervals $\left[0, s_{1}\right],\left[s_{1}, s_{2}\right], \ldots,\left[s_{k}, t_{j}\right]$, where $s_{1}, \ldots, s_{k}$ are the points where the curve $\left(\tilde{\eta}_{j}\right)$ possibly meets the end points $\eta_{i}\left(t_{i}\right)$ for some $i>j$. In this way we obtain the curves $\gamma_{1}, \ldots, \gamma_{m}$ with the property that $S=\bigcup\left[\gamma_{j}\right]$ and $\left[\gamma_{i}\right] \cap\left(\gamma_{j}\right)=\emptyset$ for all $i \neq j$.

At this point we only need to prove that every curve $\gamma_{j}$ is a straight segment. Consider any one of the curves $\gamma_{j}$, and let $x_{j}$ and $y_{j}$ be its endpoints. Define

$$
S^{\prime}:=\left(S \backslash\left(\gamma_{j}\right)\right) \cup\left[x_{j}, y_{j}\right] .
$$

Since the open curve $\left(\gamma_{j}\right)$ does not intersect any other curve of $S$, it is easy to verify that $S^{\prime}$ is compact and connected, hence $S^{\prime} \in \mathcal{S}(A)$. Since $S \in \mathcal{M}(A)$, we have

$$
\mathcal{H}^{1}(S) \leq \mathcal{H}^{1}\left(S^{\prime}\right)=\mathcal{H}^{1}(S)-\mathcal{H}^{1}\left(\left[\gamma_{j}\right]\right)+\mathcal{H}^{1}\left(\left[x_{j}, y_{j}\right]\right)
$$

hence $\mathcal{H}^{1}\left(\left[\gamma_{j}\right]\right)=\mathcal{H}^{1}\left(\left[x_{j}, y_{j}\right]\right)=\left|y_{j}-x_{j}\right|$. But the segment is the only curve with this propery, so we conclude that $\left[\gamma_{j}\right]=\left[x_{j}, y_{j}\right]$. 
LEMMA 1.3. Let $X$ be a compact connected metric space with $\mathcal{H}^{1}(X)<+\infty$. Then $X$ is pathwise connected and locally pathwise connected.

Proof. For the first claim ( $X$ pathwise connected) see [1, first rectifiability theorem]. To prove that $X$ is locally pathwise connected it is enough to prove that it is locally connected, since then we apply the first claim locally.

Let $x_{0} \in X$ and $\rho>0$ be given. We want to prove that there exists a connected neighbourhood of $x_{0}$ contained in $\overline{B_{\rho}\left(x_{0}\right)}$. Consider $X_{\rho}:=X \cap \overline{B_{2 \rho}\left(x_{0}\right)}$ and let $X_{i}$ with $i \in I$ be the connected components of $X_{\rho}$. Recall that every connected component $X_{j}$ is closed in $X_{\rho}$ and hence it is compact. Let $X_{0}$ be the connected component containing $x_{0}$. And consider the family $X_{j}$ with $j \in J, j \neq 0$, of such connected components $X_{j}$ which intersect the smaller ball $\overline{B_{\rho}\left(x_{0}\right)}$. Every $X_{j}$ is compact and does not contain $x_{0}$ so it has positive distance from $x_{0}$. If $J$ is finite there would exist a minimal distance $\varepsilon>0$ such that $X \cap B_{\varepsilon}\left(x_{0}\right)=X_{0} \cap B_{\varepsilon}\left(x_{0}\right)$ and hence $X_{0}$ would be a connected neighbourhood of $x_{0}$. On the other hand suppose that $J$ is infinite. So we have infinitely many disjoint connected sets $X_{j}$ such that $X_{j} \ni y_{j}$ with $y_{j} \in B_{\rho}\left(x_{0}\right)$. We also claim that every $X_{j}$ has a point in $\partial B_{2 \rho}\left(x_{0}\right)$. Otherwise we would conclude that $X_{j} \subseteq B_{2 \rho}\left(x_{0}\right)$ which means that $X_{j}$ is also open in $X$. And this is a contradiction with the connectedness of $X$. So for every $j \in J$ we find points $y_{j}, z_{j} \in X_{j}$ such that $d\left(x_{j}, z_{j}\right) \geq \rho$ and hence $\mathcal{H}^{1}\left(X_{j}\right) \geq \rho$. Since $J$ is infinite we would conclude that $\mathcal{H}^{1}(X)=+\infty$ which is in contradiction with the hypothesys.

Definition 1.4. Let $S \in \mathcal{S}(A)$. We say that $S$ is indecomposable, if $S \backslash A$ is connected.

THEOREM 1.5. Let $A$ be a compact and countable subset of $\mathbb{R}^{N}$. If $S \in \mathcal{M}(A), \mathcal{H}^{1}(S)<+\infty$, and $S$ is indecomposable, then

$$
S \backslash A_{0}=\bigcup_{i \in \mathbb{N}}\left[x_{i}, y_{i}\right]
$$

where $A_{0} \subseteq A, x_{i}, y_{i} \in S$ satisfy $x_{i} \neq y_{i}$ and $\left[x_{i}, y_{i}\right] \cap\left(x_{j}, y_{j}\right)=\emptyset$ for all $i \neq j, i, j \in \mathbb{N}$ and $\left(x_{i}, y_{i}\right) \cap A=\emptyset$ for all $i \in \mathbb{N}$.

Proof. Step 1. We prove that $S$ is the union of injective curves $\gamma_{i}$ such that $\left(\gamma_{i}\right) \cap\left[\gamma_{j}\right]=\emptyset$ for all $j<i$.

Let $A=\left\{a_{i}: i \in \mathbb{N}\right\}$ with $a_{i} \neq a_{j}$ for $i \neq j$ and choose $x_{0} \in S \backslash A$. Since 
$\mathcal{H}^{1}(S)<+\infty$ there exist lipschitz continuous injective curves $\eta_{j}$ : $[0,1] \rightarrow S$ such that $\eta_{j}(0)=a_{j}, \eta_{j}(1)=x_{0}$ for all $j=1,2, \ldots$. We define

$$
S_{j}:=\bigcup_{i=1}^{j}\left[\eta_{i}\right]
$$

and

$$
\begin{aligned}
& \bar{t}_{1}:=1, \\
& \bar{t}_{j}:=\min \left\{t \in[0,1]: \eta_{j}(t) \in S_{j-1}\right\}, \quad j=2,3, \ldots
\end{aligned}
$$

Then we consider the restrictions $\gamma_{j}:=\left.\eta_{j}\right|_{\left[0, \bar{t}_{j}\right]}$ and notice that $S_{j}=S_{j-1} \cup\left[\gamma_{j}\right]$ while $\left(\gamma_{i}\right) \cap\left[\gamma_{j}\right]=\emptyset$ if $j<i$. In fact every curve $\left[\gamma_{i}\right]$ can possibly meet the union $S_{i}$ of the curves $\left[\gamma_{j}\right]$ with $j<i$ only in its right extremal point $\gamma_{i}\left(\bar{t}_{i}\right)$.

Let

$$
S^{\prime}:=\bigcup_{j \in \mathbb{N}}\left[\gamma_{j}\right]=\bigcup_{j \in \mathbb{N}} S_{j} .
$$

By construction $S^{\prime}$ is bounded, connected and contains $A$. We will prove that it is also closed. Consider any sequence $x_{k} \in S^{\prime}$ such that $x_{k} \rightarrow \bar{x}$. We have to prove that $\bar{x} \in S^{\prime}$. If there exists some $j$ such that $\left[\gamma_{j}\right]$ contains $x_{k}$ for infinitely many indices $k$, then we have $\bar{x} \in\left[\gamma_{j}\right] \subseteq S^{\prime}$, and we are done. Otherwise we may assume that there exists a subsequence $x_{k_{i}}$ such that $x_{k_{i}} \in\left[\gamma_{j_{i}}\right]$ for $i=1,2, \ldots$ where $j_{i}$ is a strictly increasing sequence of indices. Now notice that

$$
\sum_{i=1}^{+\infty} \mathcal{H}^{1}\left(\left[\gamma_{j_{i}}\right]\right)=\mathcal{H}^{1}\left(\bigcup_{i=1}^{+\infty}\left[\gamma_{j_{i}}\right]\right) \leq \mathcal{H}^{1}(S)<+\infty
$$

therefore $\mathcal{H}^{1}\left(\left[\gamma_{j_{i}}\right]\right) \rightarrow 0$ as $i \rightarrow \infty$.

By construction, for all $i$ one has $\gamma_{j_{i}}(0) \in A$ and since $\left|x_{k_{i}}-\gamma_{j_{i}}(0)\right| \leq$ $\leq \mathcal{H}^{1}\left(\left[\gamma_{j_{i}}\right]\right) \rightarrow 0$ and $x_{k_{i}} \rightarrow \bar{x}$, one notices that $\gamma_{j_{i}}(0) \rightarrow \bar{x}$ as $i \rightarrow \infty$. Since $A$ is closed, it follows that $\bar{x} \in A \subseteq S^{\prime}$. Hence we have proven that $S^{\prime}$ is closed, and hence compact.

Now we are going to prove by contradiction that $S^{\prime}=S$. Otherwise since $S^{\prime} \subseteq S$ and $S^{\prime}$ is compact, there would exist $x \in S \backslash S^{\prime}$ and $r>0$ such that $S \cap B_{r}(x) \subseteq S \backslash S^{\prime}$. Thus we would have

$$
\mathcal{H}^{1}(S)-\mathcal{H}^{1}\left(S^{\prime}\right)=\mathcal{H}^{1}\left(S \backslash S^{\prime}\right) \geq \mathcal{H}^{1}\left(S \cap B_{r}(x)\right) \geq r>0
$$

and this is a contradiction to the minimality of $S$. This concludes Step 1. 
Step 2. We prove that $S$ contains no loop. We claim that there exists no continuous curve $\gamma:[0,1] \rightarrow S$ such that $\gamma(0)=\gamma(1)$ which is injective on $[0,1)$. Suppose by contradiction that such a curve $\gamma$ exists and consider the set

$$
B:=\bigcup_{j \in \mathbb{N}} \partial \gamma_{j}
$$

Notice that $B \supseteq A$ since $\gamma_{j}(0)=a_{j}$.

Since $B$ is countable, while $(\gamma)$ is not, there exists a point $x \in(\gamma) \backslash B$. We claim also that $x \notin \bar{B}$. On the contrary, there would exist a sequence of points $x_{k} \rightarrow x$ with $x_{k} \in \partial \gamma_{j_{k}}$ with $j_{k} \rightarrow \infty$. Reasoning as in the previous step, we notice that $\left|x_{k}-\gamma_{j_{k}}(0)\right| \rightarrow 0$ and since $\gamma_{j_{k}}(0) \in A$ we would conclude $x \in A \subseteq B$, which is a contradiction. So there exists a whole neighbourhood $U$ of $x$ such that $U \cap \bar{B}=\emptyset$ and in particular there would exist $i \in \mathbb{N}$, $t_{0} \in(0,1)$ and $\varepsilon>0$ such that $\gamma_{i}\left(t_{0}\right)=x$ while $\gamma_{i}(t) \notin \bar{B}$ for all $t \in I_{\varepsilon}:=\left[t_{0}-\varepsilon, t_{0}+\varepsilon\right]$. Let $\eta$ be the restriction of the curve $\gamma_{i}$ to the interval $\left[t_{0}-\varepsilon, t_{0}+\varepsilon\right]$. Since the small curve $\eta$ does not meet any curve $\left[\gamma_{j}\right]$ with $j \neq i$, and since $\eta\left(t_{0}\right) \in[\gamma]$, we conclude that $[\eta] \subseteq[\gamma]$. Then we define

$$
S^{\prime}:=S \backslash(\eta)
$$

Clearly $S^{\prime} \supseteq A$, we claim that $S^{\prime}$ is also compact. In fact if $x_{k}$ is a sequence of points in $S^{\prime}$, up to a subsequence we might suppose that $x_{k} \rightarrow x \in S$. We only have to prove that $x \notin(\eta)$. Notice that $x_{k} \in\left[\gamma_{j_{k}}\right]$ for some $j_{k} \neq i$. If there exists some $j \in \mathbb{N}$ such that $j_{k}=j$ for infinitely many values of $k \in \mathbb{N}$ we conclude that $x \in\left[\gamma_{j}\right] \subseteq S^{\prime}$. Otherwise we could find a subsequence of $j_{k}$ (not relabeled) such that $j_{k} \rightarrow \infty$. Since $\left|x_{j_{k}}-\gamma_{j_{k}}(0)\right| \leq \mathcal{H}^{1}\left(\left[\gamma_{j_{k}}\right]\right) \rightarrow 0$ we conclude that $\gamma_{j_{k}}(0) \rightarrow x$ and hence $x \in B \subseteq S^{\prime}$.

So we have proven that $S^{\prime}$ is compact. Moreover $S^{\prime}$ is pathwise connected. In fact notice that the two end-points $\partial \eta$ are connected in $S^{\prime}$ through a path in the loop $[\gamma]$. So every curve in $S$ joining points of $S^{\prime}$, can be eventually modified by means of $\gamma$ so that it does not pass through $[\eta]$.

Hence we get a contradiction to the minimality of $S$ because $\mathcal{H}^{1}(S)=\mathcal{H}^{1}\left(S^{\prime}\right)+\mathcal{H}^{1}([\eta])$ and $\mathcal{H}^{1}([\eta])>0$.

Step 3. We prove that for all $i \in \mathbb{N}$ one has $A \cap\left(\gamma_{i}\right]=\emptyset$. On the contrary there would exist a point $a \in A \cap\left(\gamma_{i}\right]$ for some index $i$. By hypothesys the set $S \backslash A$ is connected. Since $\mathcal{H}^{1}(S)<+\infty$ by Lemma 1.3 the set $S$ is locally pathwise connected. So $S \backslash A$ is connected and locally pathwise connected hence it is pathwise connected. Let $t \in\left(0, \bar{t}_{i}\right]$ be such that $\gamma_{i}(t)=a$ and let $s \in(0, t)$ be such that $x:=\gamma_{i}(s) \notin A$. Since $S \backslash A$ is pathwise connected we can find an injective curve $\gamma:[0,1] \rightarrow S \backslash A$ such that $\gamma(0)=\gamma_{i}(s)$ and $\gamma(1)=x_{0}$. Recall that $x_{0}$ was choosen in Step 1 , so that $x_{0} \notin A$ and $x_{0} \in S_{j}$ for 
all $j$. We also know that $S_{i-1}$ is connected so we can find a curve in $S_{i-1}$ joining $\gamma_{i}\left(\bar{t}_{i}\right)$ with $x_{0}$. Joining such a curve with $\gamma_{i}$ we are able to find an injective curve $\eta:[0,1] \rightarrow S$ such that $\eta(0)=\gamma_{i}(s), \eta(1 / 2)=a$ and $\eta(1)=x_{0}$. Notice that we have $\eta(0)=\gamma(0)$ and $\eta(1)=\gamma(1)$. Next we consider

$$
\begin{aligned}
& s_{1}:=\max \{t \in[0,1 / 2]: \eta(t) \in[\gamma]\} \\
& s_{2}:=\min \{t \in[1 / 2,1]: \eta(t) \in[\gamma]\}
\end{aligned}
$$

and notice that $s_{1}<1 / 2<s_{2}$ so the curve $\eta$ restricted to the interval $\left[s_{1}, s_{2}\right]$ concatenated with the curve $\gamma$ between the points $\eta\left(s_{1}\right)$ and $\eta\left(s_{2}\right)$, gives a non-trivial injective loop in $S$. And this is a contradiction to what we proved in Step 2.

Step 4. We claim that $\bar{t}_{i}>0$ for all $i \in \mathbb{N}$. We know that $\bar{t}_{1}=1$ so consider any $i>1$. Recall that $\gamma_{i}\left(\bar{t}_{i}\right) \in S_{i-1}$. In particular there exists $j<i$ such that $\gamma_{i}\left(\bar{t}_{i}\right) \in\left[\gamma_{j}\right]$. Now if we suppose by contradiction that $\bar{t}_{i}=0$ we have $\gamma_{i}\left(\bar{t}_{i}\right)=\gamma_{i}(0)=a_{i} \in A$ and hence $\left[\gamma_{j}\right] \cap A \supseteq\left\{a_{i}\right\}$. By Step 3, the only possibility is that $\gamma_{j}(0)=a_{i}$ which means that $a_{i}=a_{j}$ with $i \neq j$ which is in contradiction with the definition of the points $a_{i}$ given in Step 1.

Step 5. We split every curve $\left[\gamma_{i}\right]$ into pieces which do not contain points of $B$ in the interior. Let $i$ be a fixed index and consider the curve $\left[\gamma_{i}\right]$. Consider also the set

$$
T=\left\{t \in\left(0, \bar{t}_{i}\right): \gamma_{i}(t) \in B\right\}
$$

(recall that $B$ was defined in Step 2). We claim that $\left[0, \bar{t}_{i}\right] \backslash T$ has, at most, a countable number of connected components. If $T$ is finite the claim is trivial. Otherwise let $t_{k}$ be any sequence of points in $T$, and suppose that $t_{k} \rightarrow t$ for some $t \in\left[0, \bar{t}_{i}\right]$. We have $\gamma_{i}\left(t_{k}\right) \in \partial \gamma_{j_{k}}$ for some sequence $j_{k} \rightarrow \infty$. Hence, reasoning as we did before, $\gamma_{i}\left(t_{k}\right) \rightarrow \gamma_{i}(t) \in A$. But we have just proved that $\left(\gamma_{i}\right] \cap A=\emptyset$, hence we conclude that necessarily $t=0$. So $T$ has 0 as the only accumulation point in $\left[0, \bar{t}_{i}\right]$. This means that the sequence of points $t_{k}$ can be sorted in decreasing order, i.e. we might and shall suppose that

$$
t_{0}:=\bar{t}_{i}>t_{1}>t_{2}>\ldots>t_{j}>\ldots>0 .
$$

In particular the set $\left(0, \bar{t}_{i}\right] \backslash T$ has a countable number of connected components.

Let $m_{i} \in \mathbb{N} \cup\{+\infty\}$ be the number of connected components of $\left[0, \bar{t}_{i}\right] \backslash T$. We replace the curve $\gamma_{i}$ into $m_{i}$ pieces $\gamma_{i}^{k}=\gamma_{i\left[t_{k+1}, t_{k}\right]}$ such that

$$
\left(\gamma_{i}\right] \subseteq \bigcup_{k=0}^{m_{i}}\left[\gamma_{i}^{k}\right] \subseteq\left[\gamma_{i}\right]
$$


The family of curves $\left\{\gamma_{i}^{k}: i \in \mathbb{N}, k=0, \ldots, m_{i}\right\}$ is a countable family of curves which we could enumerate with a single index as in $\left\{\alpha_{j}: j \in \mathbb{N}\right\}$ where $\alpha_{j}=\gamma_{i}^{k}$ for some $i$ and $k$ depending on $j$. The curves $\alpha_{j}$ have the property that $\left[\alpha_{j}\right] \cap\left(\alpha_{i}\right)=\emptyset$ for all $i \neq j$ and there exists a set $A_{0} \subseteq A$ such that

$$
S \backslash A_{0}=\bigcup_{j \in \mathbb{N}}\left[\alpha_{j}\right] .
$$

Notice that it can actually happen that $A_{0}$ is not empty. For example if $A=\{1 / n: n \in \mathbb{N}\} \cup\{0\}$, then the unique minimal set $S \in \mathcal{M}(A)$ is given by $S=[0,1]$ and from the previous construction, up to renumbering, we obtain $\left[\alpha_{j}\right]=[1 /(j+1), 1 / j]$ and $A_{0}=\{0\}$.

Step 6. For all $j \in \mathbb{N}$ the set $\left(\alpha_{j}\right)$ is relatively open in $S$. Suppose by contradiction that there exists a sequence of points $x_{k} \in S \backslash\left(\alpha_{j}\right)$ such that $x_{k} \rightarrow x$ and $x \in\left(\alpha_{j}\right)$. If there are infinitely many indices $k$ such that $x_{k} \in A_{0}$, we would have $x \in \overline{A_{0}} \subseteq A$, and hence $x \notin\left(\alpha_{j}\right)$. So we might (and shall) suppose that $x_{k} \in \bigcup_{i}\left[\alpha_{i}\right]$, and hence there exists $i_{k} \in \mathbb{N}$ such that $x_{k} \in\left[\alpha_{i_{k}}\right]$ for all $k$.

Again we notice that $i_{k}$ cannot be bounded, otherwise there would exist a value $i$ such that $x_{k} \in\left[\alpha_{i}\right]$ for infinitely many values of $k$. In such a case we would find $x \in\left[\alpha_{i}\right]$ contrary to the condition $\left[\alpha_{i}\right] \cap\left(\alpha_{j}\right)=\emptyset$. Up to a subsequence, we might assume that $i_{k} \rightarrow \infty$ is strictly increasing. So reasoning as we did before, we find that $\left|\alpha_{i_{k}}(0)-x_{k}\right| \leq \mathcal{H}^{1}\left(\left[\alpha_{i_{k}}\right]\right) \rightarrow 0$. Since $\partial \alpha_{j} \subseteq B$ for every $j$, and $B$ is closed, we conclude that $x \in B$. But this is a contradiction, since by construction $\left(\alpha_{j}\right) \cap B=\emptyset$.

Step \%. The curves $\alpha_{j}$ are straight segments. Suppose the contrary, i.e. that there exists $j \in I$ such that $\left[\alpha_{j}\right] \neq\left[x_{j}, y_{j}\right]$. Then $\mathcal{H}^{1}\left(\left[x_{j}, y_{j}\right]\right)<$ $<\mathcal{H}^{1}\left(\left[\alpha_{j}\right]\right)$. Let $x_{j}, y_{j}$ be the two end-points of $\alpha_{j}$, and define

$$
\tilde{S}:=A_{0} \cup \bigcup_{i \in I \backslash\{j\}}\left[\alpha_{i}\right] \cup\left[x_{j}, y_{j}\right]=\left(S \backslash\left(\alpha_{j}\right)\right) \cup\left[x_{j}, y_{j}\right] .
$$

Now we need to prove that $\tilde{S} \in \mathcal{S}(A)$. First of all notice that $\tilde{S}$ is compact, because $\left(\alpha_{j}\right)$ is relatively open in $S$.

Then let us prove that $\tilde{S}$ is pathwise connected. Let $x, y$ be two points of $\tilde{S}$. Suppose by simplicity that $x, y \in S \backslash\left(\alpha_{j}\right)$ (the other cases being similar). Since $S$ is pathwise connected we can find a curve $\gamma$ joining $x$ with $y$. If this curve is contained in $S \backslash\left(\alpha_{j}\right)$ then it is contained in $\tilde{S}$. Otherwise we claim that the first and last points of intersection with $\left[\alpha_{j}\right]$ are $x_{j}$ and $y_{j}$. In fact suppose that $x \in\left[\alpha_{j}\right]$ is the limit of points $x_{j} \in S \backslash\left[\alpha_{j}\right]$. If there is a subsequence which lies entirely in one curve $\left[\alpha_{i}\right]$ then the limit point is in 
$\left[\alpha_{i}\right] \cap\left[\alpha_{j}\right] \subseteq\left\{x_{j}, y_{j}\right\}$. Otherwise the sequence spans infinitely many different curves $\alpha_{i}$. In this case the limit point is either a point of $A$ or a point of $A_{0}$ (as we already proved before). In both cases it cannot be a point of $\left(\alpha_{j}\right)$. So the part of curve $\gamma$ which crosses $\left(\alpha_{j}\right)$ is bounded by the two extremal points $x_{j}, y_{j}$ and can be replaced by the segment $\left[x_{j}, y_{j}\right]$. Thus $\tilde{S} \in \mathcal{S}(A)$.

On the other hand we have

$$
\begin{aligned}
\mathcal{H}^{1}(\tilde{S}) & \leq \mathcal{H}^{1}\left(S \backslash\left(\alpha_{j}\right)\right)+\mathcal{H}^{1}\left(\left[x_{j}, y_{j}\right]\right) \\
& =\mathcal{H}^{1}(S)-\mathcal{H}^{1}\left(\left[\alpha_{j}\right]\right)+\mathcal{H}^{1}\left(\left[x_{j}, y_{j}\right]\right) \\
& <\mathcal{H}^{1}(S)
\end{aligned}
$$

contrary to the minimality of $S$.

Theorem 1.6. Let $A \subseteq \mathbb{R}^{N}$ be a countable and compact set. Let $S \in \mathcal{M}(A)$. If $\mathcal{H}^{1}(S)<+\infty$ then there exists $A_{0} \subseteq A$ such that

$$
S \backslash A_{0}=\bigcup_{i \in \mathbb{N}}\left[x_{i}, y_{i}\right]
$$

where $x_{i}, y_{i} \in \mathbb{R}^{N}$ are points such that $\left[x_{i}, y_{i}\right] \cap\left(x_{j}, y_{j}\right)=\emptyset$ for all $i \neq j$, and $x_{i} \neq y_{i}$ for all $i$.

Proof. Let $C_{i}$ with $i \in I$ be the family of connected components of $S \backslash A$ and define

$$
\begin{aligned}
S_{i} & :=\overline{C_{i}} \\
A_{i} & :=S_{i} \cap A .
\end{aligned}
$$

Step 1. We claim that $\mathcal{H}^{1}\left(C_{i}\right)>0$ for all $i \in I$. Suppose by contradiction that $\mathcal{H}^{1}\left(C_{i}\right)=0$ for some $i \in I$. Since $C_{i}$ is a connected component this would mean that $C_{i}$ is a singleton $C_{i}=\{x\}$. Notice that $S \backslash A$ is locally connected because $S$ is locally connected by Lemma 1.3 , and $A$ is closed. Therefore, every connected component $C_{i}$ is relatively open in $S \backslash A$. Hence there exists $r>0$ such that $B_{r}(x) \cap(S \backslash A)=B_{r}(x) \cap S=\{x\}$ and since $S$ is connected, this implies that $S=\{x\}$ which is a trivial case.

As a consequence we find that $I$ is at most countable, otherwise we would have

$$
\mathcal{H}^{1}(C) \geq \sum_{i \in I} \mathcal{H}^{1}\left(C_{i}\right)=+\infty
$$


Step 2. We prove that (recall that $S_{i}:=\overline{C_{i}}$ )

$$
S=\overline{\bigcup_{i \in I} S_{i}}
$$

Simply notice that $S=\overline{S \backslash A}$ because $A$ is a countable set and $S \supseteq A$ is closed and connected. Hence we have

$$
S=\overline{S \backslash A}=\overline{\bigcup_{i \in I} C_{i}}=\overline{\bigcup_{i \in I} \overline{C_{i}}}
$$

Step 3. For all $i \in I$ we have $S_{i}=C_{i} \cup A_{i}$. First we will prove that $C_{i} \cup A$ is closed. Take any sequence $x_{k} \in C_{i} \cup A$ such that $x_{k} \rightarrow x$. Clearly $x \in S$ since $S$ is closed. If, by contradiction, $x \notin C_{i} \cup A$ then $x \in C_{j}$ for some $j \neq i$. Hence we would have that every neighbourhood of $x$ contains points of $C_{i}$. Hence $S \backslash A$ would not be locally connected, because every neighbourhood of $x$ intersects two different connected components of $S \backslash A$. This leads to a contradiction.

As a consequence $S_{i}=\overline{C_{i}} \subseteq C_{i} \cup A$. And by definition of $A_{i}$ we find that $S_{i} \subseteq C_{i} \cup A_{i}$. The reverse inclusion is trivial, so we conclude that $S_{i}=C_{i} \cup A_{i}$.

Step 4. We prove that $S_{i} \in \mathcal{M}\left(A_{i}\right)$. Let $S_{i}^{\prime} \in \mathcal{S}\left(A_{i}\right)$ be any competitor to $S_{i}$ and construct a competitor to $S$ as:

$$
S^{\prime}:=\left(S \backslash C_{i}\right) \cup S_{i}^{\prime}
$$

Since $S \backslash A$ is locally connected, $C_{i}$ is relatively open in $S \backslash A$ and also in $S$. Hence $S^{\prime}$ is compact.

We claim that $S^{\prime}$ is pathwise connected. Fix a point $x_{0} \in A_{i}$ and consider any point $x \in S^{\prime}$, we want to find a path connecting $x_{0}$ to $x$. We have two possibilities: either $x \in S \backslash C_{i}$ or $x \in S_{i}^{\prime}$. If $x \in S \backslash C_{i}$, since $S$ is pathwise connected we can find a continuous curve $\gamma:[0,1] \rightarrow S$ with $\gamma(0)=x$ and $\gamma(1)=x_{0}$. Then we restrict the curve to the interval $[0, \bar{t}]$ where

$$
\bar{t}:=\min \left\{t \in[0,1]: \gamma(t) \in \overline{C_{i}}\right\} .
$$

Clearly $\gamma([0, \bar{t}]) \subseteq S \backslash C_{i}$ and $y:=\gamma(\bar{t}) \in \partial_{S} C_{i}=S_{i} \backslash C_{i}$. In the previous Step we proved that $S_{i} \backslash C_{i}=A_{i}$, so we have $y \in A_{i}$. Now $y, x_{0} \in A_{i} \subseteq S_{i}^{\prime}$ and since $S_{i}^{\prime}$ is pathwise connected we can find a curve joining $y$ to $x_{0}$ in $S_{i}^{\prime}$. Hence we have found a curve joining $x$ to $x_{0}$ in $S^{\prime}$. This completes the case when $x \in S \backslash C_{i}$. The other case, namely $x \in S_{i}^{\prime}$ is easier, since $x, x_{0} \in S_{i}^{\prime}$ and $S_{i}^{\prime}$ is pathwise connected. This concludes the proof that $S^{\prime}$ is connected. 
So $S^{\prime} \in \mathcal{S}(A)$ is a competitor to $S \in \mathcal{M}(A)$ therefore $\mathcal{H}^{1}\left(S^{\prime}\right) \geq \mathcal{H}^{1}(S)$. As a consequence, we have

$$
\mathcal{H}^{1}\left(S_{i}^{\prime}\right) \geq \mathcal{H}^{1}\left(S^{\prime}\right)-\mathcal{H}^{1}\left(S \backslash C_{i}\right) \geq \mathcal{H}^{1}(S)-\mathcal{H}^{1}\left(S \backslash C_{i}\right)=H^{1}\left(C_{i}\right)=H^{1}\left(S_{i}\right) .
$$

Since this is true for every $S_{i}^{\prime} \in \mathcal{S}\left(A_{i}\right)$ we conclude that $S_{i} \in \mathcal{M}\left(A_{i}\right)$.

Step 5. Conclusion. Applying Theorem 1.5 we know that each component $S_{i}$ apart from a set $A_{0}^{i}$, is a countable union of segments which can only meet on the end points. Also we know that also the points of $A_{i}$ do not meet the interior of the segments. Hence we define

$$
A_{0}:=A \backslash \bigcup_{i \in I} S_{i}
$$

and notice that $S \backslash A_{0}=\bigcup_{i} S_{i}$ is an at most countable union of segments which can only meet on the end points.

\section{An alternative formulation.}

We could weaken the formulation of the problem to include cases when the set $A$ to be connected might have full measure. Such a formulation has been considered in [2].

Let $A$ be a compact subset of $\mathbb{R}^{n}$. We define

$\mathcal{S}^{\prime}(A):=\left\{S \subseteq \mathbb{R}^{N}: S\right.$ is compact and connected, and $S \cup A$ is connected $\}$

$\mathcal{M}^{\prime}(A):=\left\{S \in \mathcal{S}^{\prime}(A): \mathcal{H}^{1}(S) \leq \mathcal{H}^{1}\left(S^{\prime}\right), \forall S^{\prime} \in \mathcal{S}^{\prime}(A)\right\}$.

Theorem 1.1 can be easily adapted to prove existence in this formulation. Also we have:

THeorem 2.1. Suppose that $A$ is a compact subset of $\mathbb{R}^{N}$ with a finite number of connected components. Then every minimizer $S \in \mathcal{M}^{\prime}(A)$ is a finite graph with straight edges.

Proof. Let $A_{1}, \ldots, A_{m}$ be the connected components of $A$. Clearly a minimizer $S \in \mathcal{M}^{\prime}(A)$, must touch every component $A_{k}$ otherwise $S \cup A$ would not be connected. Choose a point $a_{k} \in S \cap A_{k}$ for all $k=1, \ldots, m$ and let $A^{\prime}=\left\{a_{1}, \ldots, a_{m}\right\}$. Then it is easy to show that $S \in \mathcal{M}\left(A^{\prime}\right)$. So we conclude by applying Theorem 1.6. 


\section{REFERENCES}

[1] L. Ambrosio - P. Tilli, Selected Topics on "Analysis in Metric Spaces", Quaderni della Scuola Normale Superiore, Pisa, 2000.

[2] S. Ducret - M. Troyanov, Steiner's invariant and minimal connections, Portugal. Math. (N.S.), 65 (2) (2008), pp. 237-242.

[3] G. Buttazzo - E. Oudet - E. StePanov, Optimal transportation problems with free Dirichlet regions, Progress in Nonlinear Diff. Equations and their Applications, 51 (2002), pp. 41-65.

[4] A. O. Ivanov - A. A. TUZHILIn, Minimal networks: the Steiner problem and its generalizations, CRC Press, 1994.

[5] M. Miranda, JR. - E. Paolini - E. Stepanov, On one-dimensional continua uniformly approximating planar sets, Calc. Var. Partial Differential Equations, 27 (3) (2006), pp. 287-309.

[6] E. PAOLINI - E. StEPANov, Existence and regularity for the Steiner problem, http://cvgmt.sns.it/papers/paoste09 (preprint).

[7] E. R. Reifenberg, Solution of the Plateau problem for m-dimensional surfaces of varying topological type, Acta Math., 104 (1960), pp. 1-92.

Manoscritto pervenuto in redazione il 30 aprile 2009. 